gelitten hatte und bei welchem die Untersuchung hochgradige Entzindungshyperämie des Trommelfells ergab, waren die Schmerzen sofort nach Anwendung des Karbolglyzerins beseitigt, ebenso die ganze Entr.ündung. Der Patient berichtete mir über seine Empfindung, als ob mit Zauberhand ihm seine Schmerzen plötzlich genommen worden seien. Jeder, der das Mittel in Anwendung zieht, kann über solche Erfahrungen berichten.

Besonders bei den Ohrschmerzen der Kinder sollte in allen Fällen Karbolglyzerin in Anwendung kommen, und sollte das Mittel der Hausapotheke der Kinderstube einverleibt werden. Es würde dadurch den Kindern und den Eltern manche schlaflose Nacht erspart. Da das Mittel, wio erwähnt, auch vom gesunden Ohre ertragen wird, kann kein Schaden verursacht werden.

Bei schweren Entzündungen, insbesondere wenn starke akute Nasenrachenkatarrhe oder infektiöse Mandelentzündungen bestehen, bringt das Mittel nur vorübergehend Linderung, und soll die Paracentese nicht verschoben werden. Da das Mittel die anästhesierende Wirkung hauptsächlich auf das Trommelfell ausïbt, so wird die Paracentese durch dasselbe $\mathrm{zu}$ einer schmerzlosen gemacht.

Die Instillationen mit Karbolglyzerin sind in der Tat bei den Kollegen in Berlin sehr beliebt, und habe ich viel Dank geerntet durch die Empfehlung des Mittels. Die Antwort darauf, warum sich ein Heilmittel allgemeiner Beliebtheit erfreut, brauche ich wohl nicht zu geben. Ich würde es auf das lebhafteste bedauern, wenn durch irrtümliche Auffassung vereinzelter Untersuchungsbefunde die Anwendung des so wertvollen Mittels beschränkt würde. Wer das Mittel nicht anwendet, verzichtet darauf, einer grołsen Anzahl von Ohrpatienten, insbesondere Kindern, die Schmerzen rasch und häufig dauernd $\mathrm{zu}$ beseitigen.

\title{
Die Behandlung der akuten Mittelohrentzündung mit 10 prozentigem Karbolglyzerin.
}

\section{Von Prof. Dr. Arthur Hartmann in Berlin.}

In der No. 14 dieser Wochenschrift schreibt Herr Dr. Heine aus der Universitäts-Ohrenklinik in Berlin: „Bei den praktischen Aerzten, besonders hier in Berlin, sind die Instillationen von 10\% igem Karbolglyzerin sehr beliebt, die Hartmann empfohlen hat. Sie scheinen nur einen Nachteil zu haben, den ich schon mehrfach unangenehm empfunden habe: das Trommelfellbild wird leicht verwischt. Einmal konnte ich konstatieren, daß auf der Membran ein leichter bräumlicher Aetzschorf lag."

Da langjährige Erfahrung gezeigt hat, daß bei Anwendung des $10 \%$ igen Karbolglyzerins das Trommelfellbild nicht verwischt wird nnd da das Karbolglyzerin keine ätzende Wirkung hat, so beruhen die von Herrn Heine erwähnten Nachteile nicht in Wirklichkeit, sondern nur auf einer irrtümlichen Auffassung zufällig gemachter Befunde. Gerade bei Anwendung des Karbolglyzerins, anch wenn dieselbe häufig erfolgt ist, bleiben die Einzelheiten der Trommelfellbilder vollständig bestehen, da eine Auflockerung der Epidermis nicht stattfindet. Daß das Karbolglyzerin keine ätzende Wirkung hat, geht schon daraus hervor, dals es, selbst in noch stärkerer Konzentration, auch in das gesunde Ohr ohne Schaden eingeträufelt werden kann.

Bei der akuten Mittelohrentzündung werden die Schmerzen in recht vielen Fällen sofort nach der Eintränfelung abgeschnitten und damit nicht selten anch der KrankheitsprozeB. Man findet z. B. ein Kind in teilnahmslosen Zustande, wimmernd im Bette liegen, es wird berichtet, daß dasselbe zuvor über Ohrschmerz geklagt hat, bei der Untersuchung besteht starke Rötung des Trommelfells; wird nun eine Eintrâufelung mit Karbolglyzerin gemacht, so kann man nicht selten sehen, daß3 das Kind nach wenigen Minuten frisch und gesund das Bett verläßt und seinem Spiel wieder nachgeht, ohne daß wieder Entzündungserscheinungen eintreten. - Ein Patient, dem ich das Mittel einträufelte, nachdem er 24 Stunden lang unter heftigsten Schmerzen 